\title{
Emulation of a target trial from observational data to compare effectiveness of Casirivimab/Imdevimab and Bamlanivimab/Etesevimab for early treatment of non- hospitalized patients with COVID-19
}

Mazzotta V(MD) ${ }^{12 *}{ }^{\dagger}$, Cozzi Lepri A (Prof. $)^{3 \dagger}$, Colavita F (PhD $)^{4}$, Lanini S (MD) ${ }^{1}$, Rosati R $(\mathrm{MD})^{1}$, Lalle E (PhD $)^{4}$, Mastrorosa I (MD $)^{1}$, Cimaglia C (BSc $)^{5}$, Vergori A (MD $)^{1}$, Bevilacqua Nazario (MD) ${ }^{1}$, Lapa Daniele $(\mathrm{PhD})^{4}$, Mariano Andrea (MD) ${ }^{1}$, Aurora Bettini $(\mathrm{PhD})^{4}$, Agrati C $(\mathrm{PhD})^{6}$, Piselli $\mathrm{P}(\mathrm{PhD})^{5}$, Girardi E $(\mathrm{MD})^{7}$, Castilletti $\mathrm{C}(\mathrm{PhD})^{4}$, Garbuglia AR $(\mathrm{PhD})^{4}$, Vaia F $(\mathrm{MD})^{8}$, Nicastri E $(\mathrm{MD})^{1}$, Antinori A (MD) ${ }^{1}$.

${ }^{\dagger}$ These authors have contributed equally to this work

${ }^{1}$ Clinical and Infectious Diseases Research Department, National Institute for Infectious Diseases Lazzaro Spallanzani IRCCS, Roma, Italy;

${ }^{2} \mathrm{PhD}$ course in Microbiology, Immunology, Infectious Diseases, and Transplants (MIMIT), University of Rome Tor Vergata, Rome, Italy

${ }^{3}$ Centre for Clinical Research, Epidemiology, Modelling and Evaluation (CREME), Institute for Global Health, UCL, London, UK;

${ }^{4}$ Laboratory of Virology, National Institute for Infectious Diseases Lazzaro Spallanzani IRCCS, Roma, Italy;

${ }^{5}$ Clinical Epidemiology, National Institute for Infectious Diseases Lazzaro Spallanzani IRCCS, Roma, Italy;

${ }^{6}$ Laboratory of Cellular Immunology and Pharmacology, National Institute for Infectious Diseases Lazzaro Spallanzani IRCCS, Roma, Italy;

${ }^{7}$ Scientific Direction, National Institute for Infectious Diseases Lazzaro Spallanzani IRCCS, Roma, Italy;

${ }^{8}$ Health Direction, National Institute for Infectious Diseases Lazzaro Spallanzani IRCCS, Roma, Italy.

*Corresponding Author

Valentina Mazzotta, MD

HIV/AIDS Unit

National Institute for Infectious Diseases Lazzaro Spallanzani, IRCCS

Via Portuense, 292, 00149 Rome, Italy

Tel. +390655170539 or +390655170546

Fax: +3906 55170477

e-mail: valentina.mazzotta@inmi.it

Keywords: monoclonal antibodies, SARS-COV-2, COVID-19, treatment, casirivimab/imdevimab, bamlanivimab/etesevimab. 


\begin{abstract}
Objectives

Comparative analysis between different monoclonal antibodies (mAbs) against SARS-CoV-2 are lacking. We present an emulation trial from observational data to compare effectiveness of Bamlanivimab/Etesevimab (BAM/ETE) and Casirivimab/Imdevimab (CAS/IMD) in outpatients with early mild-to-moderate COVID-19 in a real-world scenario of variants of concern (VoCs) from Alpha to Delta.
\end{abstract}

\title{
Methods
}

Allocation to treatment was subject to mAbs availability, and the measured factors were not used to determine which combination to use. Patients were followed through day 30. Viral load was measured by cycle threshold (CT) on D1 (baseline) and D7.

Primary outcome was time to COVID-19-related hospitalization or death from any cause over days 0-30. Weighted pooled logistic regression and marginal structural Cox model by inverse probability weights were used to compare BAM/ETE vs. CAS/IMD. ANCOVA was used to compare mean D7 CT values by intervention. Models were adjusted for calendar month, MASS score and VoCs. We evaluated effect measure modification by VoCs, vaccination, D1 CT levels and enrolment period.

\section{Results}

COVID19-related hospitalization or death from any cause occurred in 15 of 237 patients in the BAM/ETE group (6.3\%) and in 4 of 196 patients in the CAS/IMD group (2.0\%) (relative risk reduction [1 minus the relative risk] $72 \%$; $\mathrm{p}=0.024$ ). Subset analysis carried no evidence that the effect of the intervention was different across stratification factors. There was no evidence in viral load reduction from baseline through day 7 across the two groups $(+0.17,95 \%-1.41 ;+1.74$, $\mathrm{p}=0.83)$. Among patients who experienced primary outcome, none showed a negative RT-PCR test in nasopharingeal swab $(\mathrm{p}=0.009)$ and $82.4 \%$ showed still high viral load $(\mathrm{p}<0.001)$ on D7.

\section{Conclusions}

In a pre-Omicron epidemiologic scenario, CAS/IMD reduced risk of clinical progression of COVID-19 compared to BAM/ETE. This effect was not associated with a concomitant difference in virological response. 


\section{Introduction}

The widespread of severe acute respiratory syndrome coronavirus 2 (SARS-CoV-2) infection, causing coronavirus disease-2019 (COVID-19), continues to be a challenge for global public health. Monoclonal antibodies (mAbs) against SARS-CoV-2 have emerged as the strategy of choice for the treatment of early mild-to-moderate COVID-19 in outpatients at increased risk of clinical progression $^{1}$ and, based on data from randomized clinical trials (RCT), Bamlanivimab/Etesevimab ${ }^{2-}$ ${ }^{3}$, Casirivimab/Imdevimab ${ }^{4-5}$ and Sotrovimab ${ }^{6}$ received emergency use authorizations by the Italian Medicines Agency (AIFA) ${ }^{7}$.

In addition to RCT, data from observational cohorts ${ }^{8-9-10-11}$ have also confirmed the effectiveness of $\mathrm{mAbs}$, but comparative analysis between different options available are lacking ${ }^{12-13}$. Considering the rapid epidemiological evolution, with the emergence of new Variants of Concern (VoCs) ${ }^{14-15-16}$ that have been shown to escape ${ }^{17}$ the action of mAbs in vitro ${ }^{18-19}$ and in vivo ${ }^{20}$, real-life data about clinical impact and $\mathrm{mAb}$ comparison are useful to better clarify the scenario of currently existing drugs.

The aim of this analysis was to compare the clinical effectiveness of two mAb combinations, Bamlanivimab/Etesevimab (BAM/ETE) and Casirivimab/Imdevimab (CAS/IMD), in a real-life setting during a period in which the prevalent lineages in Italy were B.1.1.7 (Alpha) and B.1.617.2 (Delta), and B.1.529 (Omicron) was not yet circulating.

\section{Methods}

\subsection{Monoclonal antibody access program and eligible patients}

On March 2021, mAb administration program started at the National Institute for Infectious Diseases Lazzaro Spallanzani IRCCS in Rome, ruled by AIFA. Eligibility criteria included outpatients with a confirmed diagnosis of SARS-CoV-2 infection by an antigenic or molecular nasopharyngeal swab (NPS), mild or moderate symptoms of COVID-19 for 10 days or less and at least one of following risk factors for progression to severe disease: body mass index (BMI) $\geq 35$, chronical peritoneal dialysis or hemodialysis, uncontrolled or complicated diabetes mellitus, primary or secondary immunodeficiency. Subjects who were 55 years and older were also eligible if they had any cardio-cerebrovascular diseases, Chronic Obstructive Pulmonary Disease (COPD) or other chronic respiratory diseases. Patients requiring hospitalization for COVID-19 or supplemental oxygen therapy were excluded. In June $2021^{21}$, AIFA expanded use of mAbs including all patients with one of the following: 65 years and older, BMI > 30, any chronic renal impairment (including subjects undergoing peritoneal dialysis or hemodialysis), uncontrolled or complicated diabetes mellitus, any immunocompromising condition, cardio-cerebrovascular diseases (including hypertension with concomitant organ damage), COPD or other chronic respiratory diseases, chronic liver disease, hemoglobinopathies, neurodevelopmental and neurodegenerative disease. Comorbidity burden was assessed using Monoclonal Antibodies Screening Score $\left(\mathrm{MASS}^{7-22}\right.$ ) that assigned points, as follows: age $\geq 65$ ( 2 points), BMI $\geq 35$ ( 1 point), diabetes mellitus ( 2 points), chronic kidney disease ( 3 points), cardiovascular disease in a patient $\geq 55$ years ( 2 points), chronic respiratory disease in a patient $\geq 55$ years ( 2 points), hypertension in a patient $\geq 55$ years ( 1 point), and immunocompromised status ( 3 points).

All consecutive adult patient (age $>18$ ) who provided a written informed consent were included in study population. The study was approved by AIFA and National Ethics Committee.

\subsection{Monoclonal antibody administration}

Bamlanivimab/Etesevimab (700 mg/1400 mg) or Casirivimab/Imdevimab (1200 mg/1200mg) were administered by one-hour intravenous infusion and patients were observed for one hour after infusion. Allocation to treatments was pseudo-random, as a criterion of daily alternation (subject to 
drugs availability) was adopted, and as not many of the measured factors were used to determine which combination to infuse.

Patients who received Bamlanivimab as monotherapy or Sotrovimab were excluded.

\subsection{Procedures and data collection}

Outpatients visits were scheduled at baseline (D1) and at 7 (D7) and 30 (D30) days after infusion. Medical evaluation, vital signs recording, laboratory tests and reports on adverse effects were performed at each visit. If patients missed person visits, they were called by telephone to assess clinical conditions. As a real-life study, due to the high diagnostic demands related to the COVID19 pandemic, different methods were used to investigate serology and virological parameters according to the laboratory workflow and tests availability. SARS-COV-2 serology was performed by ELISA detecting anti-SARS-CoV-2 IgG, IgM, and IgA (ENZY-WELL SARS-CoV-2; DIESSE, Diagnostica Senese, Siena, Italy; positive index values $\geq 1.1$ ), or by two chemiluminescence microparticle assays (CMIA) detecting anti-Nucleoprotein and anti-Spike/RBD IgG (ARCHITECT SARS-CoV-2 IgG, and ARCHITECT SARS-CoV-2 IgG II Quantitative; Abbott Laboratories, Wiesbaden, Germany, respectively). According to the to manufacturer's instructions, for the two CMIA, Index $>1.4$ and Binding Antibody Units (BAU)/mL $\geq 7.1$ are considered positive for anti-N and anti-Spike/RBD IgG, respectively. Semi-quantitative estimation of viral load in NPS was assessed by RT-PCR using DiaSorin Simplexa ${ }^{\circledR}$ COVID-19 Direct platform (DiaSorin, Saluggia, Italy), based on cycle threshold (CT) values of S and ORF1ab genes amplification. Other RT-PCR methods used to verify the presence of SARS-CoV-2 were the Abbott m2000 RealTime System (Abbott Laboratories, Wiesbaden, Germany) and the Cobas ${ }^{\circledR}$ SARS-CoV-2 Test on the fully-automated cobas ${ }^{\circledR} 6800$ Systems (Roche Diagnostics, Rotkreuz, Switzerland).

Identification of VoCs was conducted by Sanger sequencing of the Spike coding gene on the D1 samples. During the period of Delta variant wave, the RT-PCR Simplexa ${ }^{\circledR}$ SARS-CoV-2 Variants Direct kit (DiaSorin, Saluggia, Italy).was included in the study as rapid method for the qualitative detection and differentiation of the N501Y, E484K, E484Q and L452R mutations.

\subsection{Outcomes}

The primary outcome was defined as time to hospitalization due to development of severe COVID19 or death from any cause over days 0-30. Secondary outcomes were a) time to hospitalization or death from any cause by day 30, b) time to hospitalization or death from any cause over days 3-30, c) the impact of intervention on CT values change from baseline to D7. The proportion of participants reporting adverse events were also shown as a safety endpoint.

\subsection{Statistical analysis}

Main characteristics of the participants, assessed at D1, were compared by treatment strategy using Mann-Whitney U test for continuous variables, expressed as median (IQR) or $\chi^{2}$ test or Fisher's exact test as appropriate for Categorical variables expressed as numbers and percentages. The effectiveness of the two strategies on the three outcomes was estimated and compared using a weighted pooled logistic regression model which approximates the parameters of a marginal structural Cox model by mean of inverse probability weights. Participants' follow-up accrued from the date of infusion until the date of hospitalisation, death or date of discharge. Administrative censoring was also applied at 31/12/2021 the date at which the database was frozen. Weights have been calculated using the predicted values from the pooled logistic models for the probabilities of starting BAM/ETE vs. CAS/IMD and those of censoring, respectively. Treatment was fitted as a time-fixed variable and there was no need to account for immortal time bias. Potential informative censoring was controlled for using inverse probability of censoring weights. Unweighted and 
weighted hazard ratios (HRs) with 95\% confidence intervals (CI) were shown. Assumptions regarding the underlying causal link between measured factors are pictured in Figure 1. Unweighted and weighting Kaplan-Meier estimates of the primary outcome stratified by treatment strategy were fitted. Interactions between the intervention and study population strata (type of VoC, vaccination status, level of D1 CT values and period of enrolment) were formally tested by including a multiplicative term in the marginal Cox regression model and adjusted hazard ratios (HRs) with 95\% confidence intervals (CI) were shown in a forest plot.

We compared mean CT values at D7 by treatment in analysis of covariance (ANCOVA- model adjusted for D1 CT value, month of enrolment and type of $\mathrm{VoC}$ ) and we described, using box-plots, median (IQR) of D1 and D7 CT values and its variation.

We also evaluated the associations between D7 CT response (using the cut-offs of 40 for negativity and 25 for reduced viral load) with both the intervention and the primary outcome using a chisquare and Fisher exact test as appropriate.

A descriptive analysis of self-reported side effects was also performed.

A two-sided test of less than 0.05 was considered statistically significant. All statistical analyses were performed using the SAS software, version 9.4 (Carey USA).

\section{Results}

\subsection{Study Population}

From $23^{\text {rd }}$ March 2021 to the $3^{\text {rd }}$ of December 2021, a total of 513 participants were evaluated for receiving $\mathrm{mAb}$ treatment and 433 of them (84.4\%) were included in the analysis (Figure 2). Briefly, $201(46 \%)$ were female, median age was 63 years (IQR 53-73) and 241 patients (57\%) were vaccinated. At baseline, median MASS score was 2 (IQR 0-4) and median time from symptoms onset to D1 was 5 days (IQR 3-6). Alfa (B.1.1.7) variant were identified in 71 participants (22\%), Gamma (P.1) in 25 (8\%) and Delta (B.1.617.2) in 192 (59\%); Beta (B.1.135) and Eta (B.1.525) in one participant each. Negative SARS-COV-2 serology at D1 was detected in $334(77 \%)$ participants; patients receiving CAS/IMD were more likely to have positive serology ( $34 \%$ vs $11 \%$, $\mathrm{p}<0.001)$. Among vaccinated patients, mRNA-1273 was used more frequently in patients receiving BAM/ETE, and ChAdOx1 less frequently $(\mathrm{p}=0.05)$. Overall, the participants receiving the two treatment strategies appeared to be balanced with respect to key predictors of outcomes as expected under our pseudo-random allocation design. Main characteristics according to the two treatment groups are reported in Table 1.

\subsection{Primary endpoint}

COVID19-related hospitalization or death from any cause occurred in 19 participants: 15 patients in the BAM/ETE group (6.3\%) and 4 patients in CAS/IMD group (2\%) (Table 2). Two deaths were observed, both in patients treated with BAM/ETE experiencing COVI-19 clinical progression. The majority of the events occurred before D7. In the weighted Kaplan-Meier analysis there was greater evidence for a difference in risk by intervention group ( $>10 \%$ for BAM/ETE) than in the unweighted (Figure 3A/3B). The relative hazard of COVID-19-related hospitalization or death for CAS/IMD compared to BAM/ETE was 0.28 (95\%CI 0.09-0.85; $\mathrm{p}=0.024$, Table 3A).

\subsection{Secondary endpoints}

Hospitalization or death from any cause by day 30 occurred in 29 participants (9\% in BAM/ETE vs. $4 \%$ in CAS/IMD). Overall, 18 of these secondary events (62\%) occurred within day three after infusion (Table 2).

Table 3B and 3C, showed the weighted relative hazard ratio for secondary endpoints. 


\subsection{Effect measure modification analysis}

212 The analysis by subsets carried no evidence that the effect of the intervention was different across a number of stratification variables for the primary endpoint ( $p$-values for interaction $>0.18$, Figure 4). The effect of the intervention appeared attenuated in participants with the Delta VoC but with

215 large uncertainty around these subgroup estimates.

\subsection{Analysis of covariance}

217 Median (IQR) of CT at D1 and D7 and CT increase between D1 and D7 are shown in Figure $5 \mathrm{~A} / 5 \mathrm{~B}$. We found no evidence for a difference in D7 CT mean values comparing CAS/IMD vs. BAM/ETE $(+0.17,95 \%:-1.41 ;+1.74, \mathrm{p}=0.83)$. Of note, proportions of participants with a negative SARS-COV-2 RT-PCR in NPS and with a high viral load at D7 were $26.1 \%$ in BAM/ETE vs. $25.8 \%$ in CAS/IMD ( $\mathrm{p}=0.94$ ) and $22.1 \%$ in BAM/ETE vs. $18.9 \%$ in CAS/IMD ( $\mathrm{p}=0.46$ ), respectively. Among patients who experienced primary outcome, none showed a negative SARSCOV-2 RT-PCR in NPS ( $\mathrm{p}=0.009)$ and $82.4 \%$ showed still high viral load $(\mathrm{p}<0.001)$ on D7.

\subsection{Adverse events}

Thirty-one participants reported adverse events; a breakdown of the most common adverse events reported is described in Table 4. Overall, 19 events (10\%) in the CAS/IMD group and 6 events $(3 \%)$ in the BAM/ETE group were considered related to $\mathrm{mAb}$ infusion $(\mathrm{p}=0.001)$. One patient only $(0.2 \%)$ reported severe dyspnea requiring hospitalization: this event was not considered by the investigators to be related to treatment.

\section{Discussion}

To our knowledge, this analysis is the first real-life comparison of two routinely used anti-SARSCoV-2 mAbs by a trial emulation methodology using observational data collected from outpatients with early mild-to-moderate COVID-19 and at high-risk for disease progression. In an evolving scenario of SARS-CoV-2 variants from Alpha to Delta, we found that patients receiving Casirivimab/Imdevimab had a 72\% lower risk of COVID-19 related-hospitalization or death from any cause than patients receiving Bamlanivimab/Etesevimab. The greater benefit of Casirivimab/Imdevimab was evident also excluding patients who experienced failure by day 2 (a time window in which events cannot be ascribed to lack of treatment effect), with a confirmed $78 \%$ risk reduction.

Overall, we found no evidence that the magnitude of the difference of the effect between mAb interventions on the risk of clinical outcomes was different in specific subsets of the study population. Nevertheless, the effect of Casirivimab/Imdevimab appeared to be larger in subgroups with the highest known risk of disease progression: patients with higher baseline viral load ${ }^{23}$, unvaccinated $^{24-25}$ and those enrolled before June 2021, when the target population included people with higher risk of severe outcome ${ }^{26}$.

On the other hand, there was larger uncertainty around the hazard ratio comparing interventions in the subgroup of patients infected with the Delta VoC with a 95\% CI not excluding superiority of Bamlanivimab/Etesevimab vs. Casirivimab/Imdevimab. This finding is consistent with in vitro observed retained activity of Bamlanivimab/Etesevimab ${ }^{15-27}$ against Delta variant (B.1617.2, nonAY.1/AY.2), and supports the recommended use of both Bamlanivimab/Etesevimab and Casirivimab/Imdevimab in settings of elevated Delta VoC prevalence.

To our knowledge, this is the first analysis from real-life data evaluating virological response to $\mathrm{mAb}$ treatment. It has been suggested that mAbs may act as antiviral neutralizing agents through multiple mechanisms, such as targeting free virus and virally infected cells ${ }^{28}$. Significant decrease in viral load was described in $\mathrm{RCT}^{2-4}$ for the two mAbs combinations analyzed in this study, but 
relation between virological and clinical outcomes remains uncertain ${ }^{29}$. Interestingly, our analysis showed no difference in terms of viral load reduction from D1 to D7 between Casirivimab/Imdevimab and Bamlanivimab/Etesevimab, but also displayed a very strong association between clinical and virological outcomes, suggesting that patients developing severe disease also failed in viral clearing. However, viral load was measured after the occurrence of all clinical events, so it is difficult to determine how much clinical outcome was mediated by the virological response or whether lack of virological clearance was actually a consequence of the clinical picture.

Finally, our data confirmed safety and tolerability of these two mAb combinations in a real-life unselected population.

Our analysis has some limitations. First, due to the observational nature of the study conducted in a single COVID health care center and to the lack of a randomized design, confounding bias cannot be ruled out. Further, eligibility criteria changed over time concurrently with the advent of Delta wave and with a wider use of Bamlanivimab/Etesevimab, due to available supplies. However, results were similar after controlling for MASS score in the regression models. Moreover, the lack of an early measure of CT (e.g. at D3) prevented us from investigating viral load as a potential mediator. Finally, the study was conducted before the emergence of Omicron B.1.1.529 VoC, which is going to subvert previous assessment about mAbs treatment as several in vitro studies suggest that both Bamlanivimab/Etesevimab and Casirivimab/Imdevimab did not retain a remarkable activity against Omicron ${ }^{16-30-31-32}$. Despite this, even today a proportion of illnesses and consequent hospitalizations are still due to the VoCs different from Omicron, and so knowledge of comparative data between available mAbs is still crucial for optimizing treatment in pandemic times.

Conflicts of Interests: The authors declare that the research was conducted in the absence of any commercial or financial relationships that could be construed as a potential conflict of interest.

\section{Authors' Contributions}

AA, VM conceptualized and designed the study. VM and IM wrote the protocol. VM, FC and ACL wrote the first draft of the manuscript and referred to appropriate literature. ACL was also the main responsible person for formal data analysis. AA, ACL, VM, AC, FC, CC and SL conceived, supervised the study and contributed to data interpretation. CC and PP were responsible for data curation. AV, IM, ARG, SC, EG, EN, FV revised the manuscript content, reviewed and edited the manuscript. EL, FC, CC and ARG performed all virological test. IM, AV, SL, SR enrolled participants. All authors agreed with and approved the final version of the manuscript.

Funding: The study was performed in the framework of the SARS-CoV-2 surveillance and response program implemented by the Lazio Region Health Authority. This study was supported by funds to the Istituto Nazionale per le Malattie Infettive Lazzaro Spallanzani IRCCS, Rome (Italy), from Ministero della Salute (Ricerca Finalizzata COVID-2020-12371675 and Ricerca Corrente linea 1 on emerging and reemerging infections").

\section{Acknowledgements}

The authors gratefully acknowledge nurse staff, all the patients and all members of the Outpatient clinic for MAbs administration and of laboratory:

Chiara Agrati, Andrea Antinori, Tommaso Ascoli-Bartoli, Francesco Baldini, Bartolini Barbara, Rita Bellagamba, Giulia Berno, Nazario Bevilacqua, Giulia Bonfiglio, Licia Bordi, Marta Camici, 
Rita Casetti, Concetta Castilletti, Stefania Cicalini, Claudia Cimaglia, Francesca Colavita, Angela Corpolongo, Alessandro Cozzi Lepri, Federico De Zottis, Angela D’Urso, Lavinia Fabeni, Massimo Francalancia, Marisa Fusto, Roberta Gagliardini, Letizia Giancola, Giuseppina Giannico, Emanuela Giombini, Enrico Girardi, Giulia Gramigna, Elisabetta Grilli, Susanna Grisetti, Cesare Ernesto Maria Gruber, Giuseppina Iannicelli, Eleonora Lalle, Simone Lanini, Gaetano Maffongelli, Andrea Mariano, Ilaria Mastrorosa, Giulia Matusali, Valentina Mazzotta, Silvia Meschi, Annalisa Mondi, Emanuele Nicastri, Sandrine Ottou, Claudia Palazzolo, Jessica Paulicelli, Carmela Pinnetti, Pierluca Piselli, Maria Maddalena Plazzi, Alessandra Oliva, Silvia Rosati, Martina Rueca, Alessandra

\section{Access to data}

Anonymized participant data will be made available upon reasonable requests directed to the corresponding author. Proposals will be reviewed and approved by investigator, and collaborators on the basis of scientific merit. After approval of a proposal, data can be shared through a secure online platform after signing a data access agreement.

\section{REFERENCES}

$316{ }^{1}$ COVID-19 Treatment Guidelines Panel. Coronavirus Disease 2019 (COVID-19) Treatment Guidelines. National Institutes of Health. Available at https://www.covid19treatmentguidelines.nih.gov/. [Accessed 28/12//2021].

${ }^{2}$ Gottlieb RL, Nirula A, Chen P, et al. Effect of bamlanivimab as monotherapy or in combination with etesevimab on viral load in patients with mild to moderate COVID-19: a randomized clinical trial. JAMA. 2021;325(7):632-644.

${ }^{3}$ Dougan M, Nirula A, Azizad M, et al. Bamlanivimab plus etesevimab in mild or moderate Covid19. N Engl J Med 2021;385:1382-1392.

${ }^{4}$ Weinreich DM, Sivapalasingam S, Norton T, et al. REGN- COV2, a neutralizing antibody cocktail, in outpatients with Covid-19. N Engl J Med. 2021;384(3):238-251.

${ }^{5}$ Weinreich DM, Sivapalasingam S, Norton T, et al. REGEN-COV Antibody Combination and Outcomes in Outpatients with Covid-19. N Engl J Med. 2021 Dec 2;385(23):e81. doi: 10.1056/NEJMoa2108163.

${ }^{6}$ Gupta A, Gonzalez-Rojas Y, Juarez E, et al. Early treatment for Covid-19 with SARS-CoV-2 neutralizing antibody sotrovimab. N Engl J Med 2021;385:1941-1950.

${ }^{7}$ Ministry of Health Decree. Authorisation for the temporary distribution of monoclonal antibodybased medicines for the treatment of COVID-19. Available at:

https://www.gazzettaufficiale.it/eli/id/2021/02/08/21A00788/sg.

${ }^{8}$ Ganesh R, Philpot LM, Bierle DM, et al; Monoclonal Antibody Treatment Program. Real-World Clinical Outcomes of Bamlanivimab and Casirivimab-Imdevimab among High-Risk Patients with Mild to Moderate Coronavirus Disease 2019. J Infect Dis. 2021 Jul 19:jiab377. doi: 10.1093/infdis/jiab377. Epub ahead of print. PMID: 34279629.

${ }^{9}$ Rainwater-Lovett K, T. Redd J, Stewart M, et al. Real-world Effect of Monoclonal Antibody Treatment in COVID-19 Patients in a Diverse Population in the United States medRxiv 2021.04.08.21254705; doi: https://doi.org/10.1101/2021.04.08.21254705

${ }^{10}$ Webb B, Buckel W, Vento T, et al. Real-world Effectiveness and Tolerability of Monoclonal Antibody Therapy for Ambulatory Patients With Early COVID-19, Open Forum Infectious Diseases, Volume 8, Issue 7, July 2021, ofab331, https://doi.org/10.1093/ofid/ofab331.

11 Razonable R.R., Pawlowski C, O'Horo J.C:, et al., Casirivimab-Imdevimab treatment is associated with reduced rates of hospitalization among high-risk patients with mild to moderate

346 coronavirus disease-19, EClinicalMedicine (2021), https://doi.org/10.1016/j.eclinm.2021.101102 
${ }^{12}$ K. McCreary E, Bariola R, Minnier T, et al. A Learning Health System Randomized Trial of Monoclonal Antibodies for Covid-19; medRxiv 2021.09.03.21262551; doi: https://doi.org/10.1101/2021.09.03.21262551

13 David T. Huang, Erin K. McCreary, J. Ryan Bariola, et al. Effectiveness of casirivimab and imdevimab, and sotrovimab during Delta variant surge: a prospective cohort study and comparative effectiveness randomized

trial. medRxiv 2021.12.23.21268244; doi: https://doi.org/10.1101/2021.12.23.21268244

${ }^{14}$ Hoffmann M, Arora P, Groß R, et al. SARS-CoV-2 variants B.1.351 and P.1 escape from neutralizing antibodies. Cell 2021; 184(9): 2384-2393.e12.

15 Planas D, Veyer D, Baidaliuk A, Staropoli I, Guivel- Benhassine F, Rajah MM, et al. Reduced sensitivity of SARS-CoV-2 variant Delta to antibody neutralization. Nature. 2021;596:276-80

${ }^{16}$ Cameroni E, Saliba C, Bowen JE, Rosen LE, et al. Broadly neutralizing antibodies overcome SARS-CoV-2 Omicron antigenic shift. bioRxiv [Preprint]. 2021 Dec 14:2021.12.12.472269. doi: 10.1101/2021.12.12.472269. PMID: 34931194; PMCID: PMC8687478.

${ }^{17}$ Starr TN, Greaney AJ, Dingens AS, Bloom JD. Complete map of SARS-CoV-2 RBD mutations that escape the monoclonal antibody LY-CoV555 and its cocktail with LY-CoV016. Cell Rep Med 2021; 2(4): 100255

${ }^{18}$ Wang P, Nair MS, Liu L, et al. Antibody Resistance of SARS-CoV-2 Variants B.1.351 and B.1.1.7. Nature 2021; 593: 130-135.

${ }^{19}$ Weisblum Y, Schmidt F, Zhang F, et al. Escape from neutralizing antibodies by SARS-CoV-2 spike protein variants. Elife. 2020;9

${ }^{20}$ Chen RE, Winkler ES, Case JB, et al. In vivo monoclonal antibody efficacy against SARS-CoV-2 variant strains. Nature 2021;596:103-108. doi: 10.1038/s41586-021-03720-y
21
Determina
AIFA
nella GU
n.142
del
16.06.2021. https://www.gazzettaufficiale.it/eli/gu/2021/06/16/142/sg/pdf

${ }^{22}$ Razonable RR, Aloia NCE, Anderson RJ, et al. A Framework for Outpatient Infusion of AntiSpike Monoclonal Antibodies to High-Risk Patients with Mild to Moderate Coronavirus Disease19: The Mayo Clinic Model. Mayo Clinic Proceedings.

${ }^{23}$ Dougan M, Azizad M, Mocherla B, et al. A randomized, placebo-controlled clinical trial of bamlanivimab and etesevimab together in high-risk ambulatory patients with COVID-19 and validation of the prognostic value of persistently high viral load. Clin Infect Dis. 2021 Oct 28:ciab912. doi: 10.1093/cid/ciab912. Epub ahead of print. PMID: 34718468.

${ }^{24}$ Lucy A McNamara, Ryan E Wiegand, Rachel M Burke, et al. Estimating the early impact of the US COVID-19 vaccination programme on COVID-19 cases, emergency department visits, hospital admissions, and deaths among adults aged 65 years and older: an ecological analysis of national surveillance data. The Lancet Nov 3, 2021. https://doi.org/10.1016/S0140-6736(21)02226-1 ${ }^{25}$ Tenforde MW, Self WH, Adams K, et al. Association Between mRNA Vaccination and COVID19 Hospitalization and Disease Severity. JAMA. Published online November 04, 2021. doi:10.1001/jama.2021.19499

${ }^{26}$ Bierle DM, Ganesh R, Tulledge-Scheitel S, et al. Monoclonal Antibody Treatment of Breakthrough COVID-19 in Fully Vaccinated Individuals with High-Risk Comorbidities. J Infect Dis. 2021 Nov 16:jiab570. doi: 10.1093/infdis/jiab570. Epub ahead of print. PMID: 34791298.

${ }^{27}$ Markus Hoffmann, Heike Hofmann-Winkler, Nadine Krüger, et al. SARS-CoV-2 variant B.1.617 is resistant to bamlanivimab and evades antibodies induced by infection and vaccination, Cell Reports, Volume 36, Issue 3, 2021, 109415, ISSN 2211-1247, https://doi.org/10.1016/j.celrep.2021.109415.

28 Davide Corti, Lisa A. Purcell, Gyorgy Snell, and David Veesler. Tackling COVID-19 with neutralizing monoclonal antibodies Cell 184, June 10, 2021. https://doi.org/10.1016/j.cell.2021.05.005 
${ }^{29}$ Kara W. Chew, Carlee Moser, Eric S. Daar, David A. Wohl, Jonathan Z. Li, et al. Bamlanivimab reduces nasopharyngeal SARS-CoV-2 RNA levels but not symptom duration in non-hospitalized

398 adults with COVID-19. medRxiv 2021.12.17.21268009; doi: https://doi.org/10.1101/2021.12.17.21268009. 30 Delphine Planas, Nell Saunders, Piet Maes. Considerable escape of SARS-CoV-2 variant 401 Omicron to antibody neutralization bioRxiv preprint doi: 402 https://doi.org/10.1101/2021.12.14.472630; this version posted December 15, 2021

$403{ }^{31}$ Cao Y, Wang J, Jian F, et al. Omicron escapes the majority of existing SARS-CoV-2 neutralizing 404 antibodies Nature https://doi. org/10.1038/d41586-021-03796-6 (2021).

$405{ }^{32}$ Dejnirattisai, W., Huo, J., Zhou, et al. SARS-CoV-2 Omicron-B.1.1.529 leads to widespread 406 escape from neutralizing antibody responses, Cell (2022), doi: 407 https://doi.org/10.1016/j.cell.2021.12.046. 
medRxiv preprint doi: https://doi.org/10.1101/2022.02.04.22270143; this version posted February 6, 2022. The copyright holder for this preprint (which was not certified by peer review) is the author/funder, who has granted medRxiv a license to display the preprint in perpetuity.

It is made available under a CC-BY-NC-ND 4.0 International license .

Table 1. Main characteristics at enrolment by intervention group

\begin{tabular}{|c|c|c|c|c|}
\hline \multirow{3}{*}{ Characteristics } & \multicolumn{4}{|c|}{ Regimen started } \\
\hline & BAM/ETE & CAS/IMD & $\begin{array}{c}\text { p- } \\
\text { value }\end{array}$ & Total \\
\hline & $\mathrm{N}=237$ & $\mathrm{~N}=196$ & & $\mathrm{~N}=433$ \\
\hline Gender, $n(\%)$ & & & 0.701 & \\
\hline Female & $112(47.3 \%)$ & $89(45.4 \%)$ & & $201(46.4 \%)$ \\
\hline Age, years & & & 0.798 & \\
\hline Median (IQR) & $63(53,73)$ & $62(53,74)$ & & $63(53,73)$ \\
\hline Days from sypmtoms onset to MAbs infusion & & & 0.624 & \\
\hline Median (IQR) & $5(3,6)$ & $5(3,6)$ & & $5(3,6)$ \\
\hline \multicolumn{5}{|l|}{ Comorbidities/risk factors, $n(\%)$} \\
\hline Diabetes & $33(14.0 \%)$ & $16(8.2 \%)$ & 0.056 & $49(11.4 \%)$ \\
\hline Severe obesity (BMI>35) & $33(13.9 \%)$ & $20(10.2 \%)$ & 0.240 & $53(12.2 \%)$ \\
\hline Obesity $(\mathrm{BMI}>30)$ & $72(30.4 \%)$ & $46(23.5 \%)$ & 0.108 & $118(27.3 \%)$ \\
\hline Hypertension & $97(41.5 \%)$ & $83(42.6 \%)$ & 0.817 & $180(42.0 \%)$ \\
\hline Cardiovascular disease & $42(17.9 \%)$ & $36(18.5 \%)$ & 0.891 & $78(18.2 \%)$ \\
\hline Cerebrovascular disease & $10(4.3 \%)$ & $10(5.1 \%)$ & 0.669 & $20(4.7 \%)$ \\
\hline Chronic respiratory disease & $37(15.7 \%)$ & $34(17.3 \%)$ & 0.642 & $71(16.4 \%)$ \\
\hline Renal impairment & $4(1.7 \%)$ & $1(0.5 \%)$ & 0.250 & $5(1.2 \%)$ \\
\hline Neurologic disease & $7(3.0 \%)$ & $9(4.6 \%)$ & 0.378 & $16(3.7 \%)$ \\
\hline Autoimmune disease & $24(10.2 \%)$ & $21(10.7 \%)$ & 0.865 & $45(10.4 \%)$ \\
\hline Neoplasms & $19(8.1 \%)$ & $15(7.7 \%)$ & 0.879 & $34(7.9 \%)$ \\
\hline Hematologic disease & $13(5.5 \%)$ & $13(6.7 \%)$ & 0.623 & $26(6.0 \%)$ \\
\hline Immunodeficiency & $11(4.9 \%)$ & $9(4.7 \%)$ & 0.924 & $20(4.8 \%)$ \\
\hline \multicolumn{5}{|l|}{ Vital signs at baseline } \\
\hline $\mathrm{SpO} 2$, median (IQR) & $97(96,98)$ & $97(96,98)$ & 0.289 & $97(96,98)$ \\
\hline Fever $\left(>37.5^{\circ} \mathrm{C}\right), \mathrm{n}(\%)$ & $12(5.1 \%)$ & $14(7.3 \%)$ & 0.342 & $26(6.1 \%)$ \\
\hline BMI, median (IQR) & $26.67(23.71,31.89)$ & $25.92(23.10,30.12)$ & 0.079 & $26.23(23.46,31.22)$ \\
\hline \multicolumn{5}{|l|}{ Laboratory values, median (IQR) } \\
\hline Ferritin, ng/ml & $161.5(68.00,274.0)$ & $179.0(110.0,313.0)$ & & $173.0(81.00,296.0)$ \\
\hline C-reactive protein, $\mathrm{mg} / \mathrm{dl}$ & $1.33(0.52,3.19)$ & $1.20(0.49,2.42)$ & & $1.27(0.50,2.83)$ \\
\hline Lymphocytes, /uL & $1210(850.0,1600)$ & $1160(880.0,1530)$ & & $1180(870.0,1560)$ \\
\hline \multicolumn{5}{|l|}{ Baseline SARS-COV-2 } \\
\hline Serology, $n(\%)$ & & & $<.001$ & \\
\hline Positive & $26(11.0 \%)$ & $66(33.7 \%)$ & & $92(21.2 \%)$ \\
\hline Negative & $207(87.3 \%)$ & $127(64.8 \%)$ & & $334(77.1 \%)$ \\
\hline Unknown & $4(1.7 \%)$ & $3(1.5 \%)$ & & $7(1.6 \%)$ \\
\hline Vaccination, $n(\%)$ & & & 0.077 & \\
\hline Yes (partly or fully) & $140(60.6 \%)$ & $101(52.1 \%)$ & & $241(56.7 \%)$ \\
\hline Vaccine type, $n(\%)$ & & & 0.050 & \\
\hline $\mathrm{BNT} 162 \mathrm{~b} 2$ & $75(68.8 \%)$ & $65(68.4 \%)$ & & $140(68.6 \%)$ \\
\hline mRNA-1273 & $18(16.5 \%)$ & $6(6.3 \%)$ & & $24(11.8 \%)$ \\
\hline ChAdOx1 & $8(7.3 \%)$ & $20(21.1 \%)$ & & $28(13.7 \%)$ \\
\hline Ad26.COV2.S & $8(7.3 \%)$ & $4(4.2 \%)$ & & $12(5.9 \%)$ \\
\hline Other/unknown & $31(22.1 \%)$ & $6(5.9 \%)$ & & $37(15.4 \%)$ \\
\hline SARS-COV-2 variant, $n(\%)$ & & & 0.886 & \\
\hline B.1.1.7/Alpha & $34(22.5 \%)$ & $37(21.4 \%)$ & & $71(21.9 \%)$ \\
\hline P.1/Gamma & $14(9.3 \%)$ & $11(6.4 \%)$ & & $25(7.7 \%)$ \\
\hline B.1617.2/Delta & $87(57.6 \%)$ & $105(60.7 \%)$ & & $192(59.3 \%)$ \\
\hline Other VoC & $1(0.7 \%)$ & $1(0.6 \%)$ & & $2(0.6 \%)$ \\
\hline Not done & $15(9.9 \%)$ & $19(11.0 \%)$ & & $34(10.5 \%)$ \\
\hline Baseline CT, mean $(S D)$ & $21.01 \pm 6.46$ & $20.14 \pm 6.09$ & & $20.56 \pm 6.28$ \\
\hline MASS score, median (IQR) & $2(0,4)$ & $2(0,3)$ & 0.104 & $2(0,4)$ \\
\hline Enrolled after June 2021, n(\%) & $172(72.6 \%)$ & $127(64.8 \%)$ & 0.082 & $299(69.1 \%)$ \\
\hline
\end{tabular}


medRxiv preprint doi: https://doi.org/10.1101/2022.02.04.22270143; this version posted February 6, 2022. The copyright holder for this preprint (which was not certified by peer review) is the author/funder, who has granted medRxiv a license to display the preprint in perpetuity.

It is made available under a CC-BY-NC-ND 4.0 International license .

\section{Table 2. Main outcomes by intervention groups}

413

414

415

\section{A. Main outcomes by intervention group}

\begin{tabular}{ccccc}
\multicolumn{4}{c}{ Regimen started } \\
Outcomes & BAM/ETE & CAS/IMD & $\begin{array}{c}\text { p- } \\
\text { value }^{*}\end{array}$ & Total \\
& $\mathrm{N}=237$ & $\mathrm{~N}=196$ & & $\mathrm{~N}=433$ \\
Primary Outcome $^{\mathrm{a}}, \boldsymbol{n}(\%)$ & $15(6.3 \%)$ & $4(2.0 \%)$ & 0.030 & $19(4.4 \%)$ \\
${ }_{\text {Ist }}$ Secondary Outcome $^{\mathrm{b}}, \boldsymbol{n}(\%)$ & $21(8.9 \%)$ & $8(4.1 \%)$ & 0.048 & $29(6.7 \%)$ \\
${ }^{2 n d}$ Secondary Outcome $^{\mathrm{c}}, \boldsymbol{n}(\%)$ & $9(3.8 \%)$ & $2(1.0 \%)$ & 0.068 & $11(2.5 \%)$
\end{tabular}

Notes: BAM/ETE, bamlanivimab/etesevimab; CAS/IMD, casirivimab/imdevimab

${ }^{\text {a }}$ Patients with COVID-19 related hospitalization or death for any cause over days 0-30

${ }^{b}$ Patients with hospitalization or death for any cause over days 0-30

${ }^{c}$ Patients with hospitalization or death for any cause over days 3-30

*Chi-square 
Table 3. Relative Hazard from fitting a marginal model using BAM/ETE as the comparator group for primary (A) and secondary endpoints (B-C).

420

421

422

423

424

425

426

427

428

429

430

431

432

433

434

435

436

\section{A. RH from fitting a marginal model for primary endpoint}

BAM/ETE

CAS/IMD

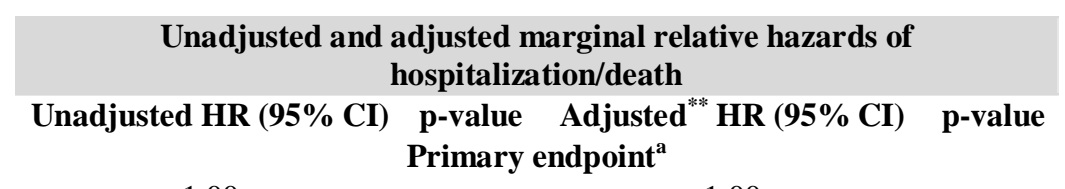

1.00

$0.32(0.10,0.95)$

0.041

$0.28(0.09,0.85)$

0.024

\section{B. RH from fitting a marginal model for 1st secondary endpoint}

BAM/ETE

CAS/IMD

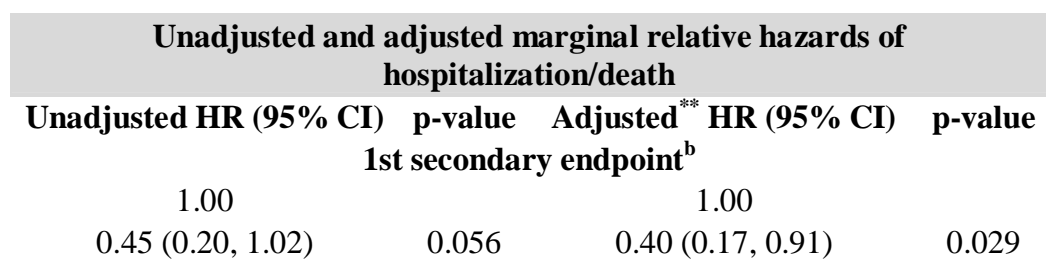

C. RH from fitting a marginal model for 2nd secondary endpoint

Notes: BAM/ETE, bamlanivimab/etesevimab; CAS/IMD, casirivimab/imdevimab

${ }^{\mathrm{a}}$ Patients with COVID-19 related hospitalization or death for any cause over days 0-30

${ }^{\mathrm{b}}$ Patients with hospitalization or death for any cause over days 0-30

${ }^{c}$ Patients with hospitalization or death for any cause over days 3-30

**adjusted for calendar month, MASS score and VoCs

437 
medRxiv preprint doi: https://doi.org/10.1101/2022.02.04.22270143; this version posted February 6, 2022. The copyright holder for this preprint (which was not certified by peer review) is the author/funder, who has granted medRxiv a license to display the preprint in perpetuity.

It is made available under a CC-BY-NC-ND 4.0 International license .

Table 4. Proportions of participants reporting specific adverse events by intervention group

\begin{tabular}{lcccc} 
& & & \\
& & Regimen started & \\
\multicolumn{1}{c}{ Characteristics } & BAM/ETE & CAS/IMD & $\begin{array}{c}\text { p- } \\
\text { value }\end{array}$ & Total \\
& $\mathrm{N}=237$ & $\mathrm{~N}=196$ & & $\mathrm{~N}=433$ \\
Adverse events, $\boldsymbol{n ( \% )}$ & & & 0.064 & \\
Mild & $8(3.4 \%)$ & $15(7.7 \%)$ & & $23(5.3 \%)$ \\
Moderate & $0(0.0 \%)$ & $7(3.6 \%)$ & & $7(1.6 \%)$ \\
Severe & $1(0.4 \%)$ & $0(0.0 \%)$ & & $1(0.2 \%)$ \\
Drug related & $6(2.5 \%)$ & $19(9.7 \%)$ & 0.001 & $25(5.8 \%)$ \\
Individual events, $\boldsymbol{n}(\%)$ & & & & \\
Arthomyalgia & $1(0.4 \%)$ & $2(1.0 \%)$ & 0.455 & $3(0.7 \%)$ \\
Asthenia & $0(0.0 \%)$ & $0(0.0 \%)$ & & $0(0.0 \%)$ \\
Chills & $0(0.0 \%)$ & $3(1.5 \%)$ & 0.056 & $3(0.7 \%)$ \\
Diarrhea & $1(0.4 \%)$ & $1(0.5 \%)$ & 0.893 & $2(0.5 \%)$ \\
Fever & $4(1.7 \%)$ & $17(8.7 \%)$ & $<.001$ & $21(4.8 \%)$ \\
Nausea & $0(0.0 \%)$ & $4(2.0 \%)$ & 0.027 & $4(0.9 \%)$ \\
Sight decrease & $0(0.0 \%)$ & $1(0.5 \%)$ & 0.271 & $1(0.2 \%)$ \\
Headache & $0(0.0 \%)$ & $1(0.5 \%)$ & 0.271 & $1(0.2 \%)$ \\
Dyspnea & $1(11.1 \%)$ & $1(4.5 \%)$ & 0.506 & $2(6.5 \%)$ \\
Abdominal pain & $1(11.1 \%)$ & $0(0.0 \%)$ & 0.118 & $1(3.2 \%)$ \\
Hematoma & $0(0.0 \%)$ & $0(0.0 \%)$ & & $0(0.0 \%)$ \\
Paraesthesia & $1(0.4 \%)$ & $0(0.0 \%)$ & 0.363 & $1(0.2 \%)$ \\
Itch & $0(0.0 \%)$ & $0(0.0 \%)$ & & $0(0.0 \%)$ \\
Skin rash & $0(0.0 \%)$ & $2(1.0 \%)$ & 0.119 & $2(0.5 \%)$ \\
Dizziness & $0(0.0 \%)$ & $0(0.0 \%)$ & & $0(0.0 \%)$ \\
Vomit & $0(0.0 \%)$ & $1(0.5 \%)$ & 0.271 & $1(0.2 \%)$
\end{tabular}

*Chi-square or Mann-Whitney test as appropriate

Notes BAM/ETE, bamlanivimab/etesevimab; CAS/IMD, casirivimab/imdevimab; IQR, interquartile range; $\mathrm{BMI}$, body mass index; $\mathrm{SpO} 2$, peripheral oxygen saturation 


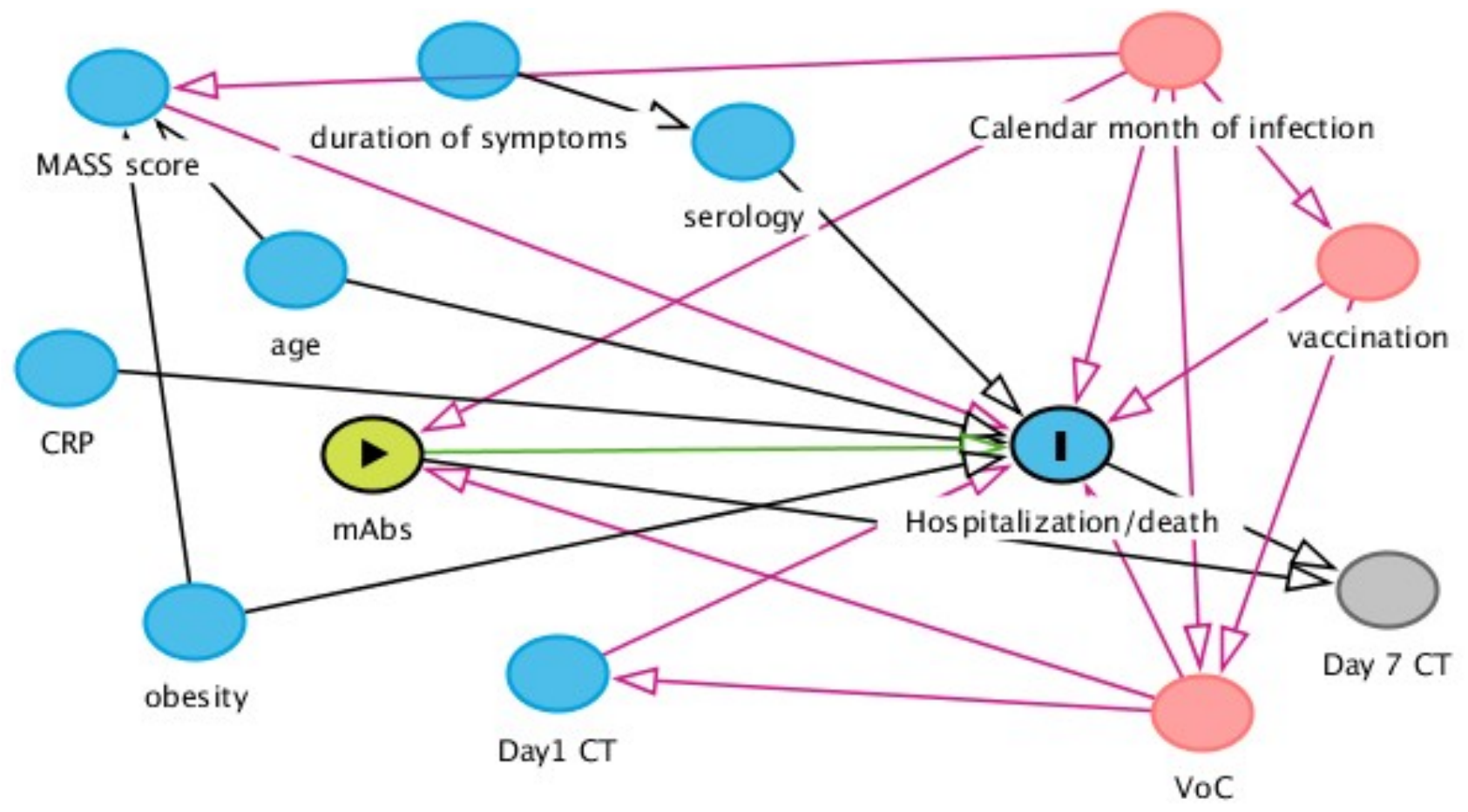

Allocation to BAM/ETE or CAS/IMD was pseudo random as not many of the measured factors were used to determine which combination to use.

According to our assumptions, month of infusion and type of $\mathrm{VoC}$ were identified as main time-fixed confounders of our comparison of interest. MASS score was an important predictor and used in some of the models to increase efficiency.

MASS score assigned points, as follows: age $\geq 65$ ( 2 points), BMI $\geq 35$ ( 1 point), diabetes mellitus ( 2 points), chronic kidney disease ( 3 points), cardiovascular disease in a patient $\geq 55$ years ( 2 points), chronic respiratory disease in a patient $\geq 55$ years ( 2 points), hypertension in a patient $\geq 55$ years ( 1 point), and immunocompromised status ( 3 points). 
medRxiv preprint doi: https://doi.org/10.1101/2022.02.04.22270143; this version posted February 6, 2022. The copyright holder for this preprint (which was not certified by peer review) is the author/funder, who has granted medRxiv a license to display the preprint in perpetuity.

Figure 2. Study flow chart is made available under a CC-BY-NC-ND 4.0 International license.

\section{Enrollment}

Assessed for eligibility $(n=513)$

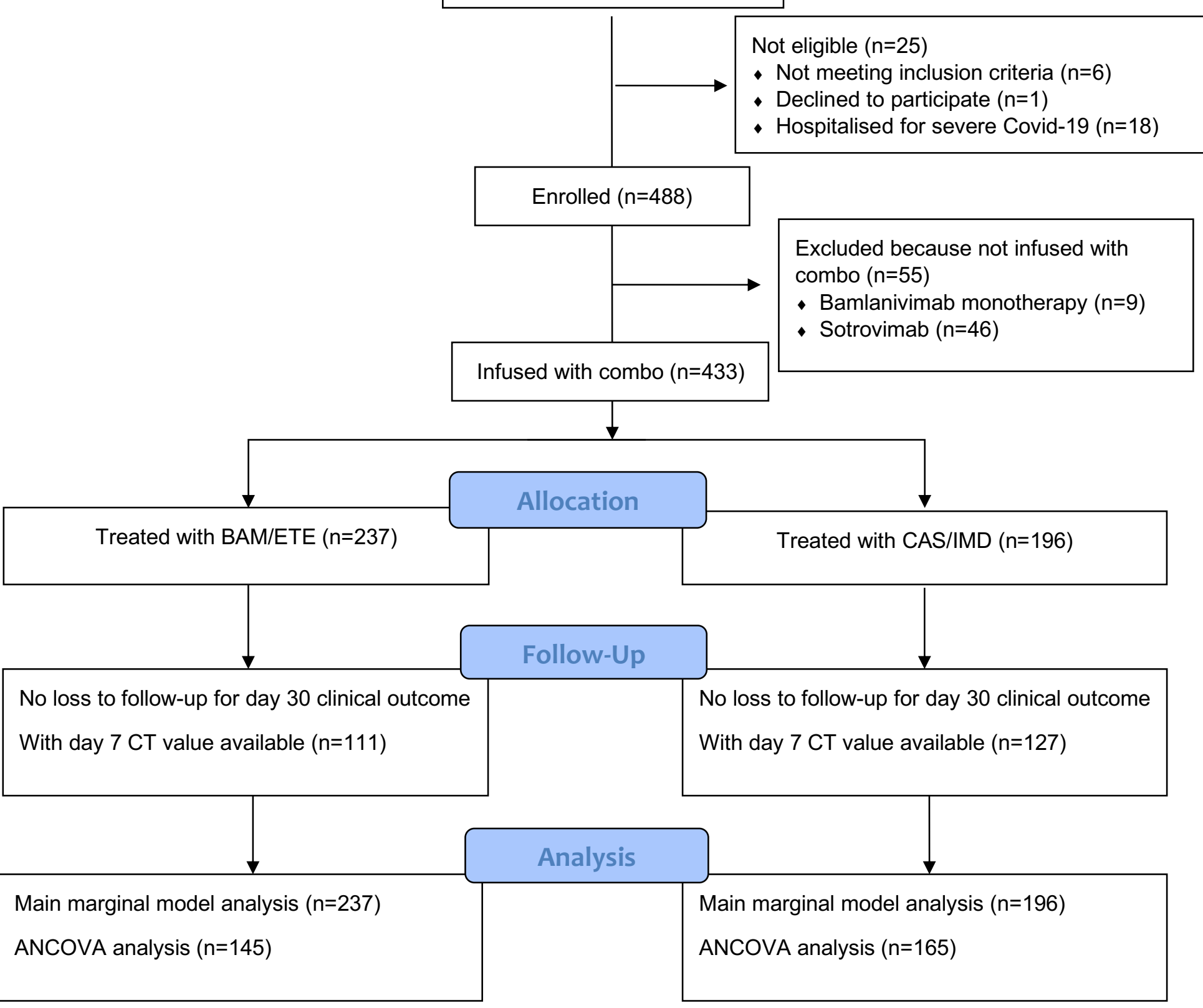


medRxiv preprint doi: https://doi.org/10.1101/2022.02.04.22270143; this version posted February 6, 2022. The copyright holder for this preprint (which was not certified by peer review) is the author/funder, who has granted medRxiv a license to display the preprint in perpetuity.

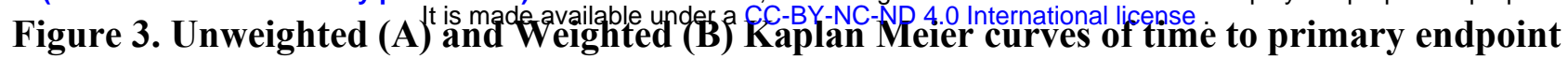
by intervention group -weights include month of enrolment and type of $\mathrm{VoC}$

A

Primary outcome: hospitalisation due to COVID-19 or death

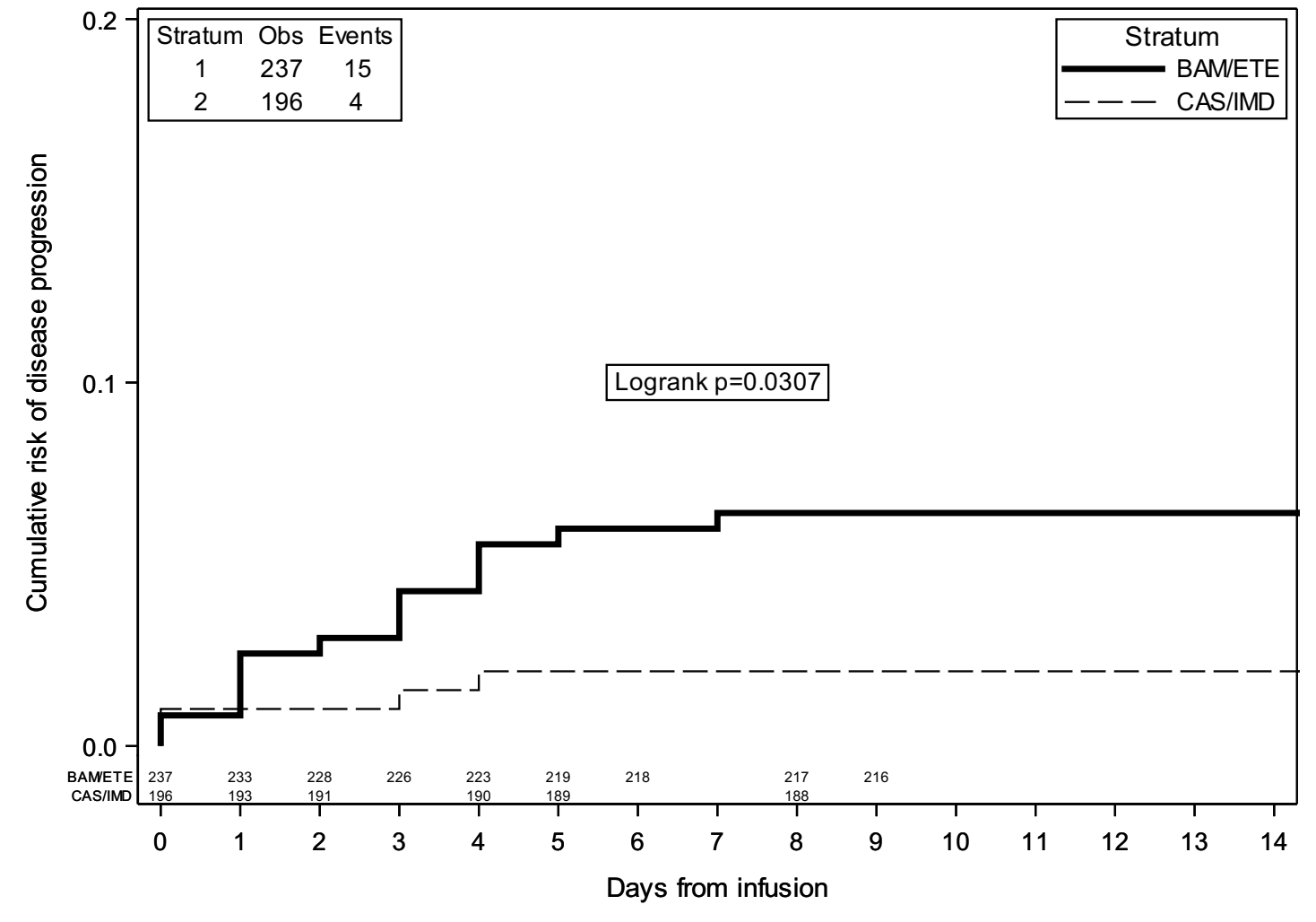

B

Primary outcome: hospitalisation due to COVID-19 or death

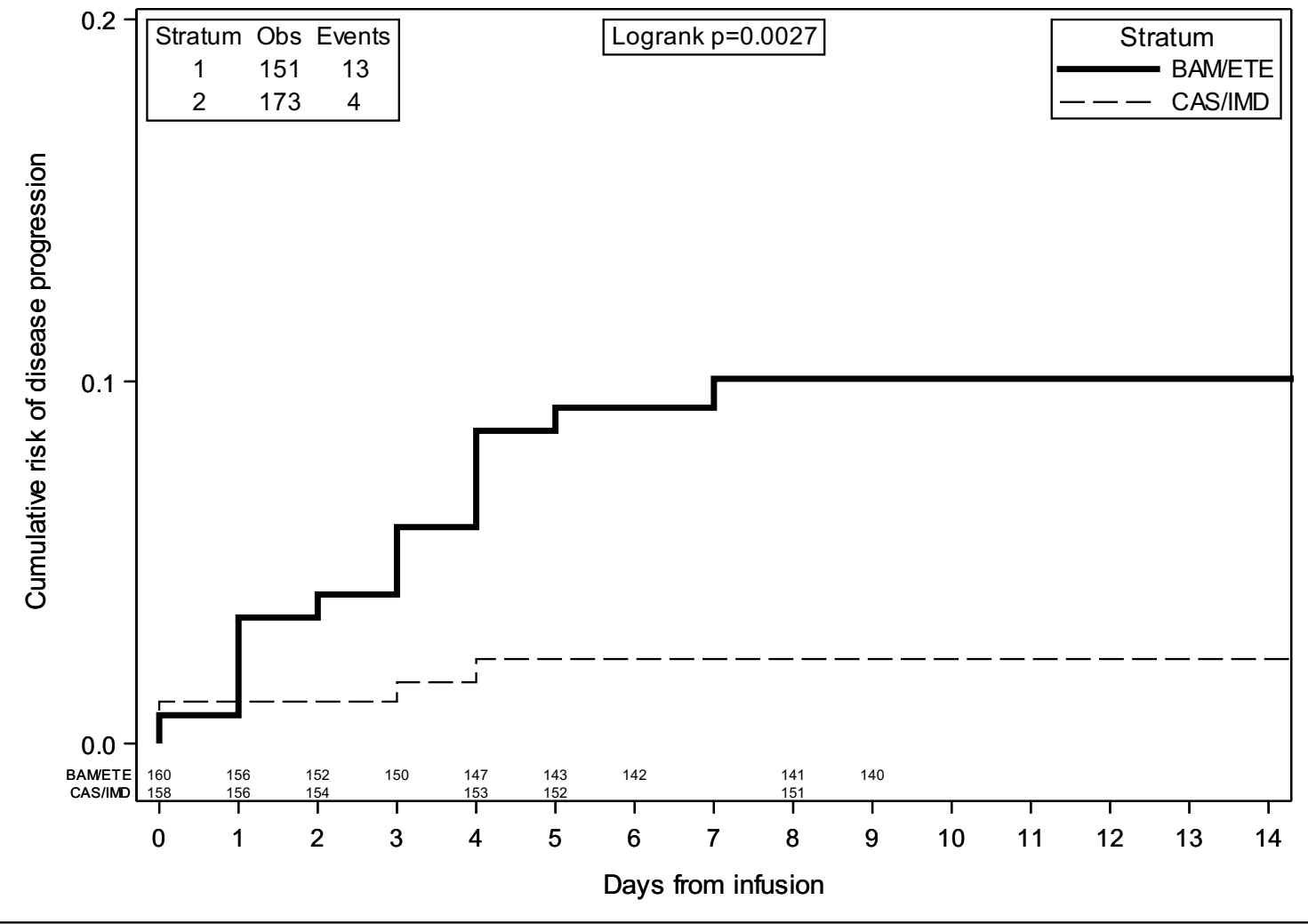




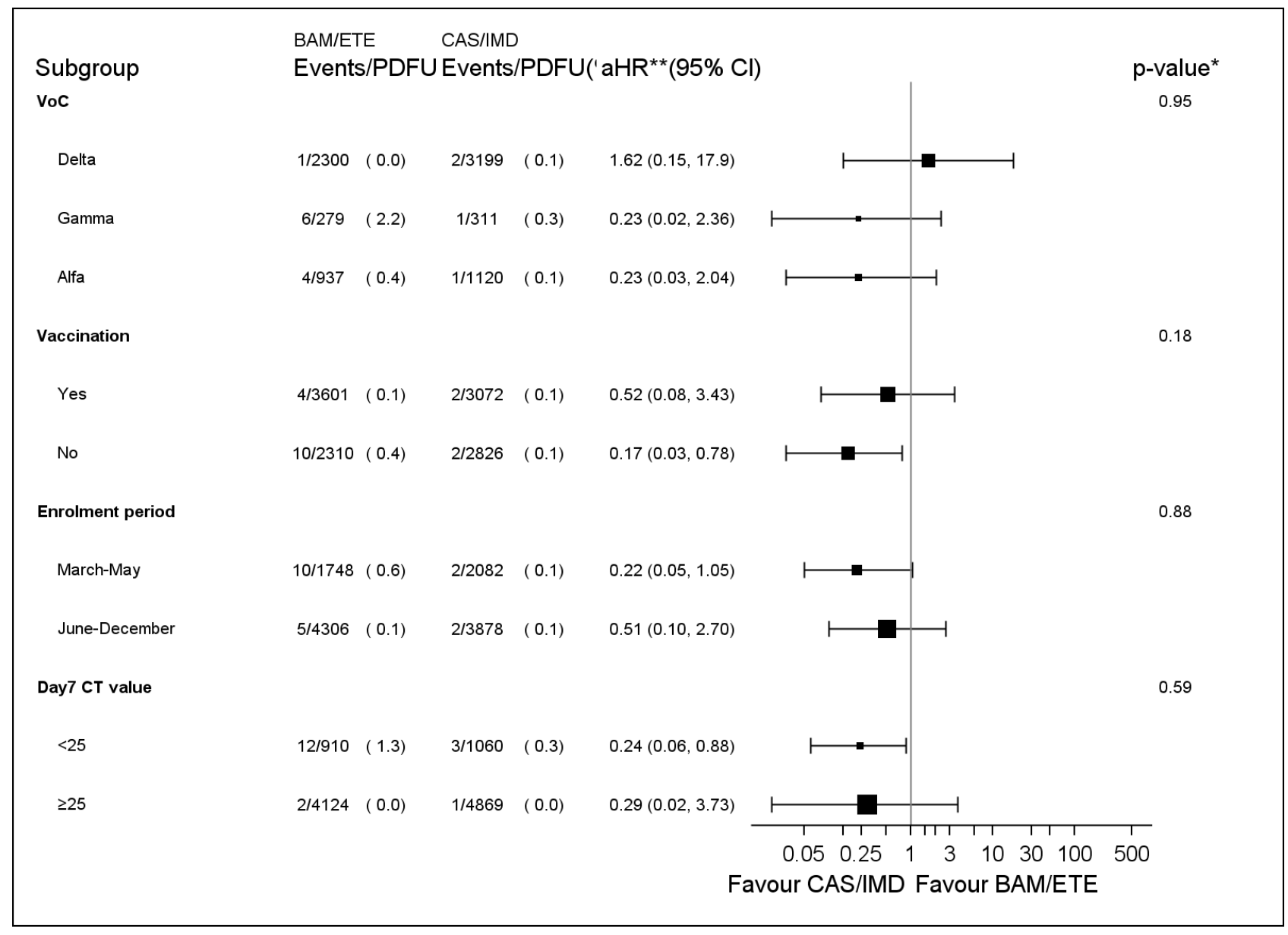

*p-value corresponds to the test for interaction between intervention and each subgroup unadjusted for multiplicity

**aHR1- (vaccination and CT strata) adjusted for calendar month and VoC;

**aHR2 - (VoC stratum) adjusted for MASS score because of a positivity issue leading to abnormally high weights in the stratum of participants infected with the Delta VoC;

**aHR3 - (enrolment period stratum) adjusted for MASS score and VoC

$\mathrm{PDFU}=$ person days of follow-up 
A

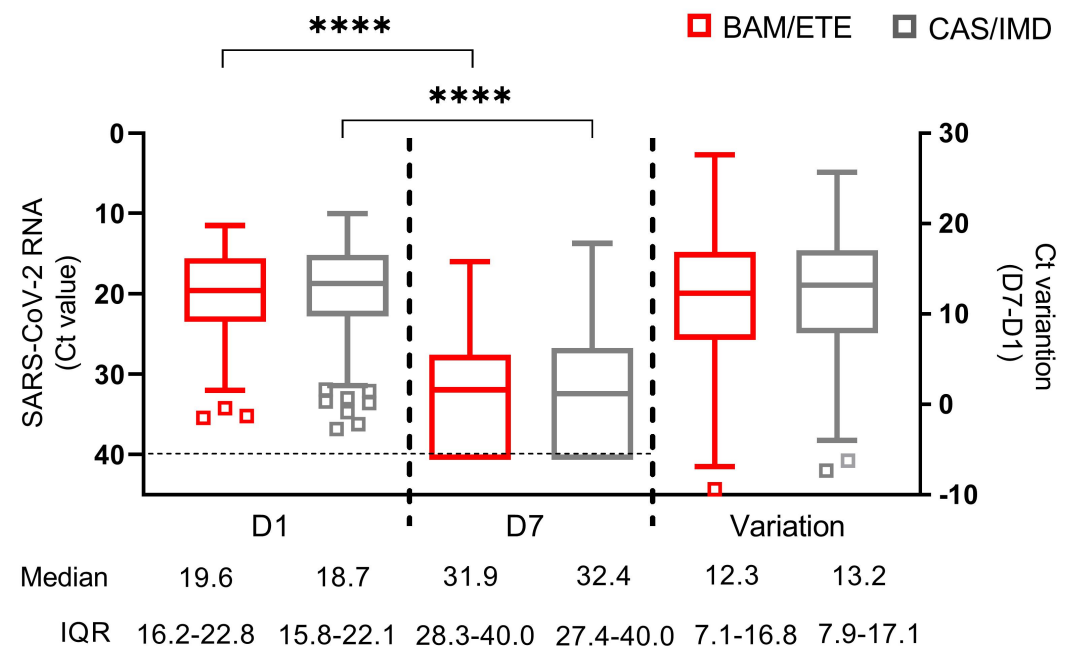

B

C
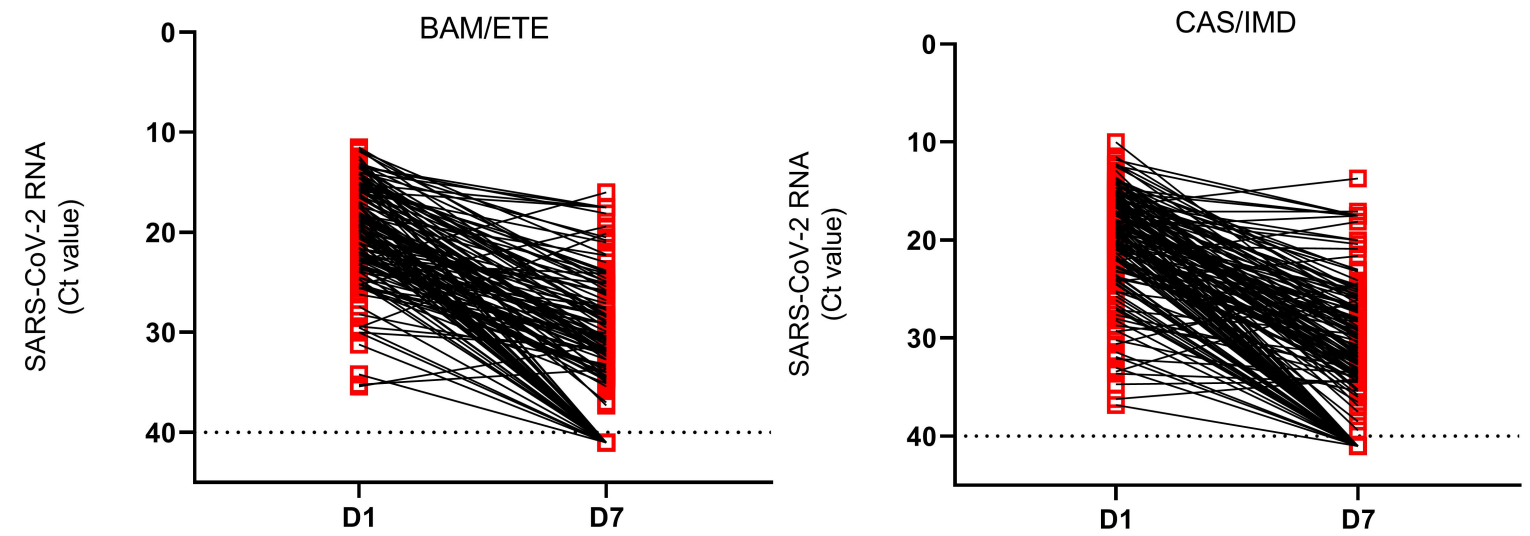

(A) Box-and-whiskers plot showing the comparison of viral loads detected at D1 and D7 in patients treated with BAM/ETE ( $\mathrm{n}=170$ and 154 , respectively) or CAS/IMD ( $\mathrm{n}=183$ and 174, respectively), and the variation of RNA levels observed between the two time-points by intervention (BAM/ETE, $\mathrm{n}=145$; CAS/IMD, $\mathrm{n}=165$ ). Viral RNA levels are expressed as CT of Orflab gene amplification. Median CT values and IQR are shown. Statistical analysis of the comparisons between treatment groups was performed by Mann-Whitney test: D1, $\mathrm{p}=0.29$; D7, $\mathrm{p}=0.87$; and Variation, $\mathrm{p}=0.71$. CT values at D1 and D7 within each group were compared using paired Wilcoxon sign-rank test, $* * * * p<0.0001$.

(B-C) Spaghetti-plot showing the Orflab CT values measured at D1 and D7 in each patient treated with BAM/ETE $(n=145$, B) or CAS/IMD $(n=165, C)$.

Horizontal dashed line represents the limit of detection (CT: 40.0 ), values $\geq 40$ are considered negative. 